\title{
Repetitive low-frequency stimulation reduces epileptiform synchronization in limbic neuronal networks
}

\author{
G. D'Arcangelo, ${ }^{a}$ G. Panuccio, ${ }^{\mathrm{b}}$ V. Tancredi, ${ }^{\mathrm{a}}$ and M. Avoli ${ }^{\mathrm{b}, \mathrm{c}, \mathrm{d}, *}$ \\ a Dipartimento di Neuroscienze, Università degli Studi di Roma 'Tor Vergata', 00173, Roma, Italy \\ b Dipartimento di Fisiologia Umana e Farmacologia, Università degli Studi di Roma 'La Sapienza', 00100, Roma, Italy \\ ${ }^{\mathrm{c}}$ Montreal Neurological Institute and Departments of Neurology and Neurosurgery and Physiology, McGill University, \\ 3801 University Street, Room 768, Montreal, Quebec, Canada H3A 2B4 \\ dIRCCS Neuromed, 86077 Pozzilli (Is), Italy
}

Received 23 June 2004; revised 30 September 2004; accepted 23 November 2004

Available online 16 February 2005

Deep-brain electrical or transcranial magnetic stimulation may represent a therapeutic tool for controlling seizures in patients presenting with epileptic disorders resistant to antiepileptic drugs. In keeping with this clinical evidence, we have reported that repetitive electrical stimuli delivered at approximately $1 \mathrm{~Hz}$ in mouse hippocampus-entorhinal cortex (EC) slices depress the EC ability to generate ictal activity induced by the application of 4-aminopyridine (4AP) or $\mathrm{Mg}^{2+}$-free medium (Barbarosie, M., Avoli, M., 1997. CA3-driven hippocampalentorhinal loop controls rather than sustains in vitro limbic seizures. J. Neurosci. 17, 9308-9314.). Here, we confirmed a similar control mechanism in rat brain slices analyzed with field potential recordings during 4AP $(50 \mu \mathrm{M})$ treatment. In addition, we used intrinsic optical signal (IOS) recordings to quantify the intensity and spatial characteristics of this inhibitory influence. IOSs reflect the changes in light transmittance throughout the entire extent of the slice, and are thus reliable markers of limbic network epileptiform synchronization. First, we found that in the presence of $4 \mathrm{AP}$, the IOS increases, induced by a train of electrical stimuli $(10 \mathrm{~Hz}$ for $1 \mathrm{~s})$ or by recurrent, single-shock stimulation delivered at $0.05 \mathrm{~Hz}$ in the deep EC layers, are reduced in intensity and area size by low-frequency $(1 \mathrm{~Hz})$, repetitive stimulation of the subiculum; these effects were observed in all limbic areas contained in the slice. Second, by testing the effects induced by repetitive subicular stimulation at $0.2-10 \mathrm{~Hz}$, we identified maximal efficacy when repetitive stimuli are delivered at $1 \mathrm{~Hz}$. Finally, we discovered that similar, but slightly less pronounced, inhibitory effects occur when repetitive stimuli at $1 \mathrm{~Hz}$ are delivered in the EC, suggesting that the reduction of IOSs seen during repetitive stimulation is pathway dependent as well as activity dependent. Thus, the activation of limbic networks at low frequency reduces the intensity and spatial extent of the IOS changes that accompany ictal synchronization in an in vitro slice preparation. This conclusion supports the view that repetitive stimulation may

* Corresponding author. Montreal Neurological Institute and Departments of Neurology and Neurosurgery and Physiology, McGill University, 3801 University Street, Room 768, Montreal, Quebec, Canada H3A 2B4. Fax: +1 5143988106 .

E-mail address: massimo.avoli@mcgill.ca (M. Avoli).

Available online on ScienceDirect (www.sciencedirect.com). represent a potential therapeutic tool for controlling seizures in patients with pharmacoresistant epileptic disorders.

(C) 2004 Elsevier Inc. All rights reserved.

Keywords: Entorhinal cortex; 4-aminopyridine; Slice preparation; Repetitive electrical stimulation; Subiculum

\section{Introduction}

Over the last few years, several attempts have been made to use deep-brain electrical (Velasco et al., 2000a,b, 2001; Vonck et al., 2002; Yamamoto et al., 2002) or transcranial magnetic stimulation (Menkes and Gruenthal, 2000; Tergau et al., 1999) to abate seizures in patients presenting with epileptic disorders resistant to antiepileptic drugs, including mesial temporal lobe epilepsy (MTLE). These stimulating procedures have been often proved to be effective in reducing and/or abolishing both interictal and ictal discharges. However, these experiments included a limited number of patients. Moreover, two fundamental points for establishing a solid scientific rationale are as yet missing: first, which brain structure should be stimulated in a specific epileptic disorder; second, what patterns of stimulation (e.g., frequency or intensity) have to be used.

By analyzing the mechanisms of ictogenesis in combined mouse entorhinal cortex (EC)-hippocampus slices superfused with 4aminopyridine (4AP) or $\mathrm{Mg}^{2+}$-free medium, we have found that CA3-driven interictal activity inhibits the EC propensity to generate ictal discharges resembling the electrographic limbic seizures seen in MTLE patients (Barbarosie and Avoli, 1997; Barbarosie et al., 2002). Moreover, we discovered that when this inhibitory control is removed by cutting the Schaffer collaterals (which represent the main output pathway for CA3 pyramidal cells), electrical stimuli delivered in the subiculum at frequencies similar to those of CA3driven interictal discharges are capable of depressing EC ictogenesis. Similar evidence has recently been obtained in the EC of 
rat brain slices in which repetitive stimuli were delivered in the lateral-basolateral nucleus of the amygdala (Benini et al., 2003). Overall, these data indicate that repetitive electrical stimuli of parahippocampal networks (which were surgically separated from the CA3 outputs) can mimic the inhibitory control exerted on EC ictogenesis by CA3-driven output activity. In addition, they suggest that this in vitro experimental model may be used for analyzing the mechanisms by which repetitive stimulation controls the generation and spread of ictal activity within limbic neuronal networks.

Here, we have further characterized the effects induced by repetitive electrical stimulation on ictal synchronization in vitro by using standard field potential recordings along with intrinsic optical signals (IOSs) in rat hippocampus-EC slices superfused with 4AP-containing medium. IOSs recorded in vitro reflect the changes in lightscattering properties and absorption that are secondary to cell swelling leading to alterations in the extracellular space volume (Andrew and MacVicar, 1994; Buchheim et al., 1999, 2000; Holthoff and Witte, 1996; Hochman et al., 1995; MacVicar and Hochman, 1991; Meierkord et al., 1997; see, for review, Andrew et al., 1999). Moreover, excitatory synaptic transmission represents a prerequisite for IOS generation (D'Arcangelo et al., 2001; Dodt et al., 1996; MacVicar and Hochman, 1991). Although it is known that the time course of IOSs is much slower than what obtained with field potential recordings (cf. Dodt et al., 1996; Buchheim et al., 2000; Holthoff and Witte, 1996; Hochman et al., 1995; Meierkord et al., 1997), this method can provide valuable quantitative information on the intensity and spatial characteristics of the inhibitory influence that is induced by repetitive electrical stimuli over the entire extent of the slice.

Since spontaneous epileptiform activity is uncommon in the submerged slice preparation (D'Arcangelo et al., 2001; Meierkord et al., 1997), discharges resembling ictal epileptiform events in field potential recordings were elicited during $4 \mathrm{AP}$ application by focal extracellular stimuli. In particular, we were interested to answer the following questions: (i) which is the extent of the inhibitory influence exerted by repetitive stimulation on ictogenesis in different limbic areas that are comprised in the brain slice? (ii) which are the repetitive stimulation frequencies that are most effective in reducing ictogenesis? and (iii) are these inhibitory effects input and/or activity dependent?

\section{Methods}

Rat brain slices were obtained following standard procedures (Benini et al., 2003; D'Arcangelo et al., 2001). In brief, animals were decapitated under halothane anesthesia, the brain was removed, and it was placed in cold oxygenated artificial cerebrospinal fluid (ACSF). Horizontal slices (450 $\mu \mathrm{m}$ thick), including the EC and the hippocampus proper, were cut with a vibratome along a horizontal plane of the brain that was tilted by approximately $10^{\circ}$ along a posterosuperior-anteroinferior plane passing between the lateral olfactory tract and the brain stem base. Slices were then transferred to a submerged tissue chamber that was mounted on the stage of an upright microscope (Axioskop FS, Zeiss) to visualize IOSs. Slices were placed under a nylon filament grid to prevent dislocation, and were superfused with oxygenated $\left(95 \% \mathrm{O}_{2}, 5 \% \mathrm{CO}_{2}\right)$ ACSF at $32-$ $34^{\circ} \mathrm{C}$. ACSF composition was (in $\mathrm{mM}$ ): $\mathrm{NaCl} \mathrm{124,} \mathrm{KCl} 2, \mathrm{KH}_{2} \mathrm{PO}_{4}$ $1.25, \mathrm{MgSO}_{4} 2, \mathrm{CaCl}_{2} 2, \mathrm{NaHCO}_{3} 26$, and glucose $10.4 \mathrm{AP}(50 \mu \mathrm{M})$ was bath applied. Chemicals were acquired from Sigma (St. Louis, MO, USA). The Schaffer collateral pathway was cut in all slices at the beginning of the experimental session by using a razor blade that was mounted on a micromanipulator (cf. Barbarosie and Avoli, 1997).

Field potential recordings were made with ACSF-filled glass pipettes (tip diameter $<10 \mu \mathrm{m}$; resistance $=5-10 \mathrm{M} \Omega$ ) that were positioned in the middle to deep layers of the EC. Electrical stimuli were delivered through bipolar, stainless steel electrodes that were placed in the subiculum and/or in the deep layers of the EC (most often in its lateral portion). Short trains of electrical stimuli $(10 \mathrm{~Hz}$ for $1 \mathrm{~s}$ at intervals $\geq 200 \mathrm{~s}$ ) or recurrent, single-shock stimuli at $0.05 \mathrm{~Hz}$ were applied in the deep layers of the EC at strengths (50$80 \mu \mathrm{A}$, up to $120 \mu \mathrm{s}$ ) that were sufficient to elicit robust epileptiform responses mirrored by IOS changes through the extent of the slice. In contrast, repetitive stimuli $(0.2-10 \mathrm{~Hz})$ were applied in the subicular area (and in some cases, in the EC) at strengths of 10-20 $\mu \mathrm{A}$, up to $100 \mu \mathrm{s}$; these intensities were unable to elicit any sustained epileptiform activity. Throughout the text, when using field potential recordings, we arbitrarily defined as ictal-like responses the synchronous events that had duration $\geq 4 \mathrm{~s}$.

To visualize IOSs, combined hippocampus-EC slices were illuminated with a dark-field condensor and examined with a $2.5 \times$ objective; the illumination light was filtered with a band-pass interference filter $(750 \pm 50 \mathrm{~nm})$. To detect IOS changes, analog contrast enhancement, background subtraction, and a fivefold digital enhancement were applied on line to video images that were obtained from an infrared video camera (C2400-07, Hamamatsu Photonics, Hamamatsu City, Japan). Images were processed with a real-time digital video image enhancement system (Argus 20, Hamamatsu Photonics). Averaged dark-field images obtained under basal condition were digitized at 16 bits, stored on a personal computer, and then subtracted in real time from the incoming image before and at successive times after single-shock or tetanic electrical stimulation. Images were acquired before (background) and during electrical stimulation with a temporal resolution of 2 images/s and were displayed in pseudocolors, assigning a color look-up table to the relative pixel values (cf. D'Arcangelo et al., 2001). Data were further analyzed off-line with the software IAS2000 (Delta Sistemi, Roma, Italy) to quantify both the intensity and the area of the IOS changes in windows that covered different regions of the slice as detailed in Fig. 2A. IOS intensity was measured calculating the difference between pixel intensity in each region of interest and it was expressed as percentage of the digital intensity of the control (background) image of that series, using the following formula: $\left[\left(T_{t}-T_{0}\right) / T_{0}\right] \times 100$ (where $T_{0}$ corresponds to the initial image of the series considered as background, and $T_{t}$ is the image acquired at a given time $t$ after the end of the stimulus). Measurements throughout the text are expressed as mean \pm SEM and $n$ represents the number of slices studied. Data were compared with the Student's $t$ test or the ANOVA test and were considered significantly different if $P<0.05$. The Spearman's rank correlation test was used to calculate the correlation coefficient between the reduction in IOS intensity and the decrease in area size occurring during repetitive stimulation.

\section{Results}

\section{Field potential recordings}

Fig. 1A illustrates the field potential activity recorded from the middle layers of the EC in the presence of 4AP, following singleshock stimulation of the EC deep layers at $0.01 \mathrm{~Hz}$ before (Control) and during repetitive stimuli delivered in the subiculum 
A
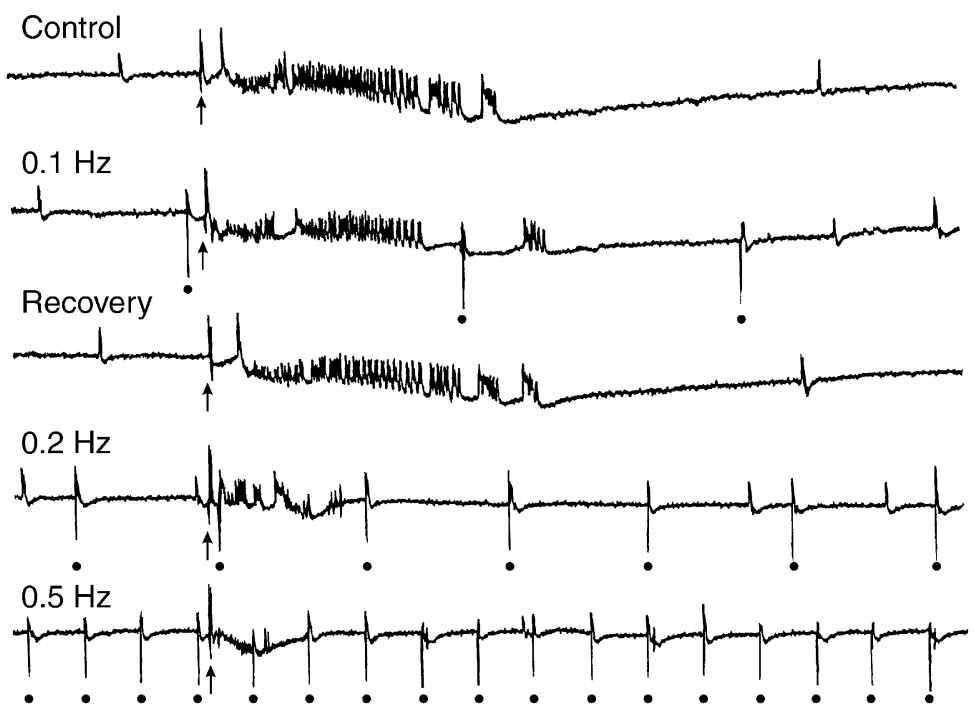

$1 \mathrm{~Hz}$

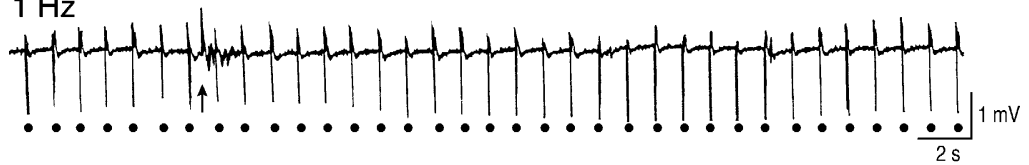

B

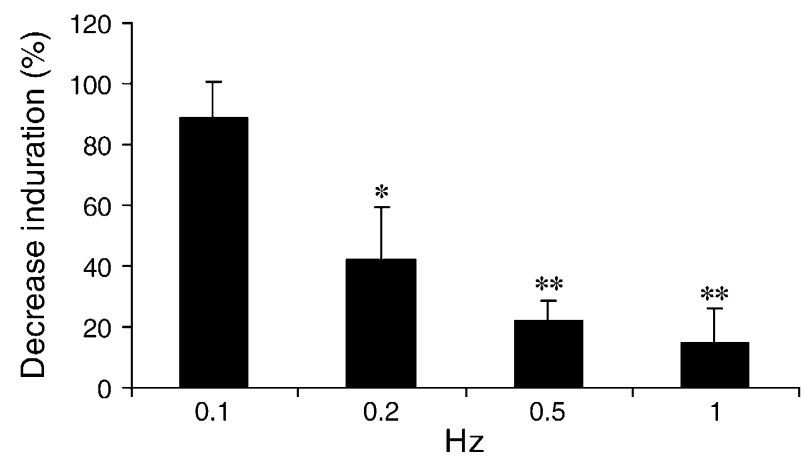

Fig. 1. Repetitive subicular stimulation reduces ictal-like discharges generated during 4AP application by EC networks in response to local single-shock stimulation of the deep layers at $0.01 \mathrm{~Hz}$. (A) Field potential activity recorded from the EC middle layers under control conditions and during repetitive stimuli delivered in subiculum at frequencies ranging from 0.1 to $1 \mathrm{~Hz}$. Stimuli delivered in the EC and subiculum are identified by arrows and filled circles, respectively. Note also that the strength of the EC and subiculum stimuli was adjusted to elicit sustained epileptiform discharges lasting up to $15 \mathrm{~s}$ and short $(<180 \mathrm{~ms})$ population bursts, respectively. The recovery sample was obtained 2 min after termination of subicular stimulation at $0.1 \mathrm{~Hz}$. (B) Normalized reduction of the duration of the epileptiform responses induced by EC stimulation in 8 slices that were studied during different subicular stimulation protocols. Levels of significance: ${ }^{*} P<0.05$ and ${ }^{* *} P<0.01$.

at frequencies ranging from 0.1 to $1 \mathrm{~Hz}$. As mentioned in the Methods section, the strength of the EC (arrows) and subiculum (filled circles) stimuli was adjusted to elicit sustained ictal-like epileptiform discharges (up to $15 \mathrm{~s}$ in duration) and short-lasting (up to $180 \mathrm{~ms}$ ) population bursts, respectively. Moreover, the ECinduced ictal responses were monitored: (i) under control conditions (i.e., in the absence of subicular stimulation) for periods of approximately $10 \mathrm{~min}$, and (ii) during repetitive stimuli of the subiculum at different rates for time spans of 4-10 min. In all cases ( $n=11$ slices), the stimulation rate was changed after a period of recovery (i.e., no subicular stimulation) lasting for at least 6 min.

In line with previous findings obtained by analyzing the spontaneous ictal discharges disclosed by bath application of 4AP (Barbarosie and Avoli, 1997; Barbarosie et al., 2002), repetitive subicular stimuli were able to reduce the duration of the ictal-like responses induced by EC stimulation. These inhibitory effects persisted throughout the stimulating procedure (up to $15 \mathrm{~min}$ ), and recovered upon its termination within approximately $1.5 \mathrm{~min}$. Overall, we observed significant reduction in the duration of the ictal-like responses during repetitive subicular stimulation set at $0.2 \mathrm{~Hz}(P<0.05)$ in 8 out of 11 slices, while prolonged stimulusinduced epileptiform discharges disappeared in all experiments when stimuli were delivered at 0.5 or $1 \mathrm{~Hz}(P<0.01$ in both cases). The normalized reduction in the duration of the epileptiform responses recorded from 8 slices during these different stimulation protocols is summarized in Fig. 1B.

\section{Effects induced by repetitive subicular stimulation at $1 \mathrm{~Hz}$ on stimulus-induced IOS changes}

Next, we used IOS recordings to identify the intensity and spatial characteristics of the epileptiform synchronization seen during 4AP application following focal activation of EC networks as well as the changes induced on these IOSs by repetitive stimuli delivered at 
$1 \mathrm{~Hz}$ in the subiculum. This frequency was selected as it appeared to be the most effective in reducing the ictal-like responses analyzed with field potential recordings (Fig. 1; cf., also Benini et al., 2003).

In a first series of experiments $(n=9)$, we studied the effects exerted by $1 \mathrm{~Hz}$ subicular stimulation on the IOS responses induced by a brief train of stimuli ( $10 \mathrm{~Hz}$ for $1 \mathrm{~s})$ applied in the EC deep layers every $5 \mathrm{~min}$. As shown in Fig. 2 (EC train row), this type of stimulating protocol evoked IOSs that appeared first in the EC (7 out of 9 experiments; arrow in the 5-s frame) or in the subiculum ( 2 out of 9 slices; not illustrated) and propagated afterwards to the hippocampus proper as well as to the perirhinal cortex (10-s frames). Similar IOS patterns have been described in detail in a previous study performed in our laboratories (D'Arcangelo et al., 2001). In contrast, continuous $1-\mathrm{Hz}$ subicular stimulation at low strength failed in inducing any detectable IOS changes (Fig. 2, Subiculum at $1 \mathrm{~Hz}$ row). Moreover, when the EC train was delivered during continuous $1 \mathrm{~Hz}$ stimulation of the subiculum at $1 \mathrm{~Hz}$, the IOSs were significantly reduced in the EC and virtually absent in the hippocampus proper (Fig. 2, EC train + Subiculum at $1 \mathrm{~Hz}$ row).

The percentage of the IOS increases and the spatial extent of the IOS changes observed in different regions of the slice following EC train stimulation in control and during repetitive $1-\mathrm{Hz}$ subicular stimulation are summarized for 9 experiments in Figs. 3A and B. These data are also plotted as percentage of IOS inhibition and of area reduction in Figs. $3 \mathrm{C}$ and $\mathrm{D}$, respectively. Overall, these findings indicate that IOSs were significantly diminished in all limbic areas during stimulation of the subiculum at $1 \mathrm{~Hz}$, but with maximal inhibition in the hippocampal CA3 area.

In a second series of experiments $(n=10)$, we analyzed the effects induced by repetitive subicular stimuli on the in IOS changes that accompany the epileptiform responses induced by single-shock stimuli delivered in the EC deep layers every $20 \mathrm{~s}$ (i.e., at $0.05 \mathrm{~Hz}$ ). Under control conditions, this procedure evoked after the first stimulus of each series (1-s frame) a relatively small increase in IOSs that was initially identified in EC (7 out of 10 slices) or in subiculum (remaining experiments), while further stimuli caused IOS changes that were more intense and propagated to the hippocampus proper and the perirhinal cortex (Fig. 4, EC at $0.05 \mathrm{~Hz}$ row). Hence, this protocol demonstrated that additional stimulations caused IOS changes of increasing intensity that involved progressively broader limbic areas.

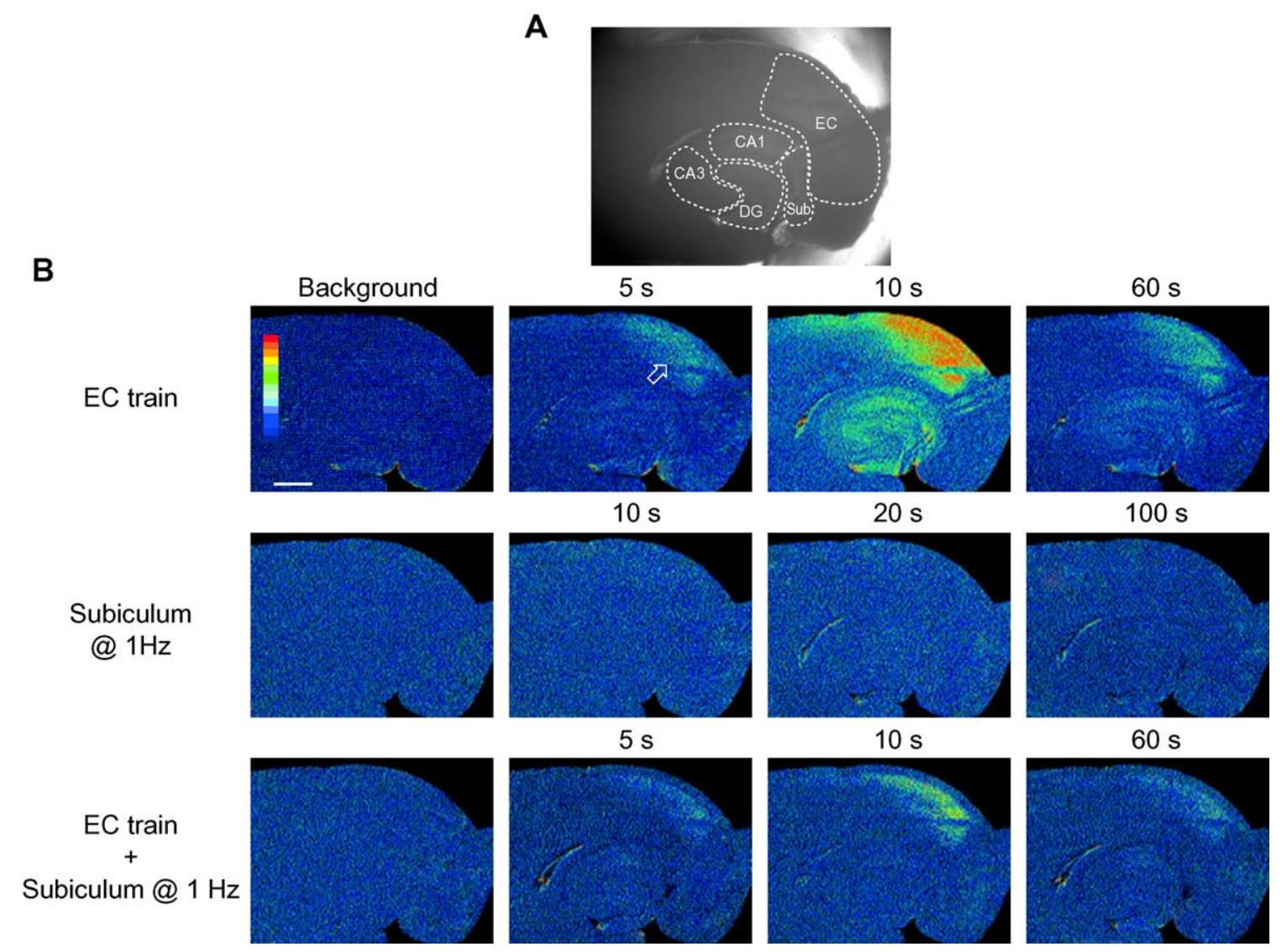

Fig. 2. (A) Infrared-darkfield image of a combined hippocampus-EC slice showing the boundaries of the different areas where IOS changes were measured. (B) IOS changes elicited: (i) by a brief stimulus train (10 Hz for $1 \mathrm{~s}$ ) delivered in the EC deep layers (upper row), (ii) by repetitive subicular stimuli at $1 \mathrm{~Hz}$ (middle row), and (iii) by pairing the two stimulation protocols (lower row). IOS frames in the upper and lower rows were obtained before (Background) and at three different times after the brief stimulus train in the EC, while frames in the middle row represent successive frames before (background) and during repetitive stimuli delivered in the subiculum at $1 \mathrm{~Hz}$ for $3 \mathrm{~min}(10-20 \mu \mathrm{A}, 0.1 \mathrm{~ms})$; note that the time shown on the top of each frame in the middle row indicates the lapse from the onset of repetitive stimulation. Note also that the samples shown in the lower row were obtained during continuous repetitive stimulation at $1 \mathrm{~Hz}$ that was started $60 \mathrm{~s}$ before the background sample. Stimulation of the EC in this experiment causes IOSs that appear first in the EC (arrow in 5-s frame) and then increase in intensity to propagate to the hippocampus proper as well as to the perirhinal cortex (10-s frame). In contrast, continuous 1 -Hz subicular stimulation at low strength does not induce any appreciable IOS change. However, when the EC train is delivered during continuous subicular stimulation at $1 \mathrm{~Hz}$, IOSs are reduced in the EC and virtually absent in the hippocampus proper. Scale bar in the background frame in the upper row $=2.5 \mathrm{~mm}$. 
A

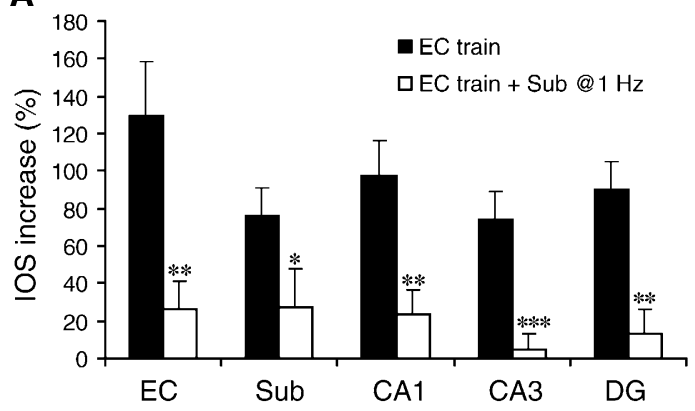

C

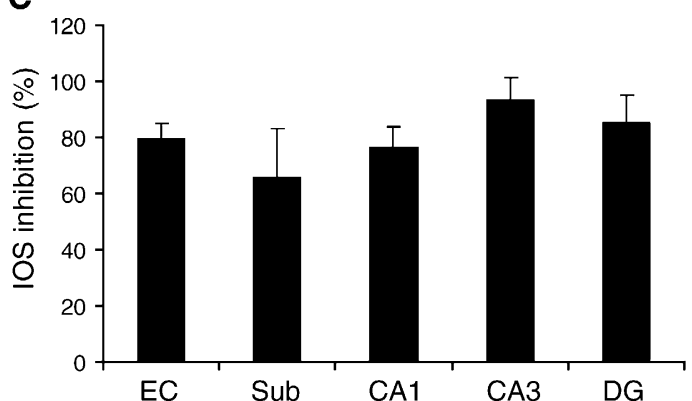

B

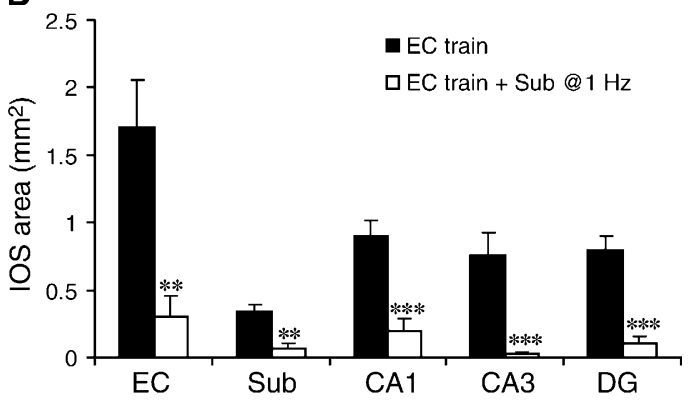

D

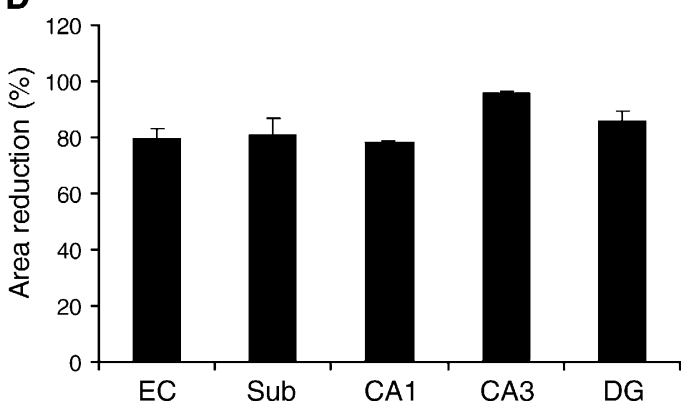

Fig. 3. Quantification of the IOS changes elicited in 9 slices treated with 4AP by brief stimulus trains ( $10 \mathrm{~Hz}$ for $1 \mathrm{~s})$ delivered in the EC deep layers under control conditions and during repetitive subicular stimuli at $1 \mathrm{~Hz}$. (A and B) Measurements of the IOS changes in terms of \% peak increases and area size in different regions of the brain slice; values were measured under control conditions and during repetitive subicular stimulation at $1 \mathrm{~Hz}, 10 \mathrm{~s}$ after the stimulus train delivered in the EC. (C and D) Inhibition of the peak IOS increases and of the size of their area exerted by 1-Hz subicular stimulation in the same experiments shown in A and B. Note that maximal inhibition of IOSs occurs in CA3. Levels of significance: ${ }^{*} P<0.05 ;{ }^{* *} P<0.01$; and ${ }^{* * *} P<0.001$.

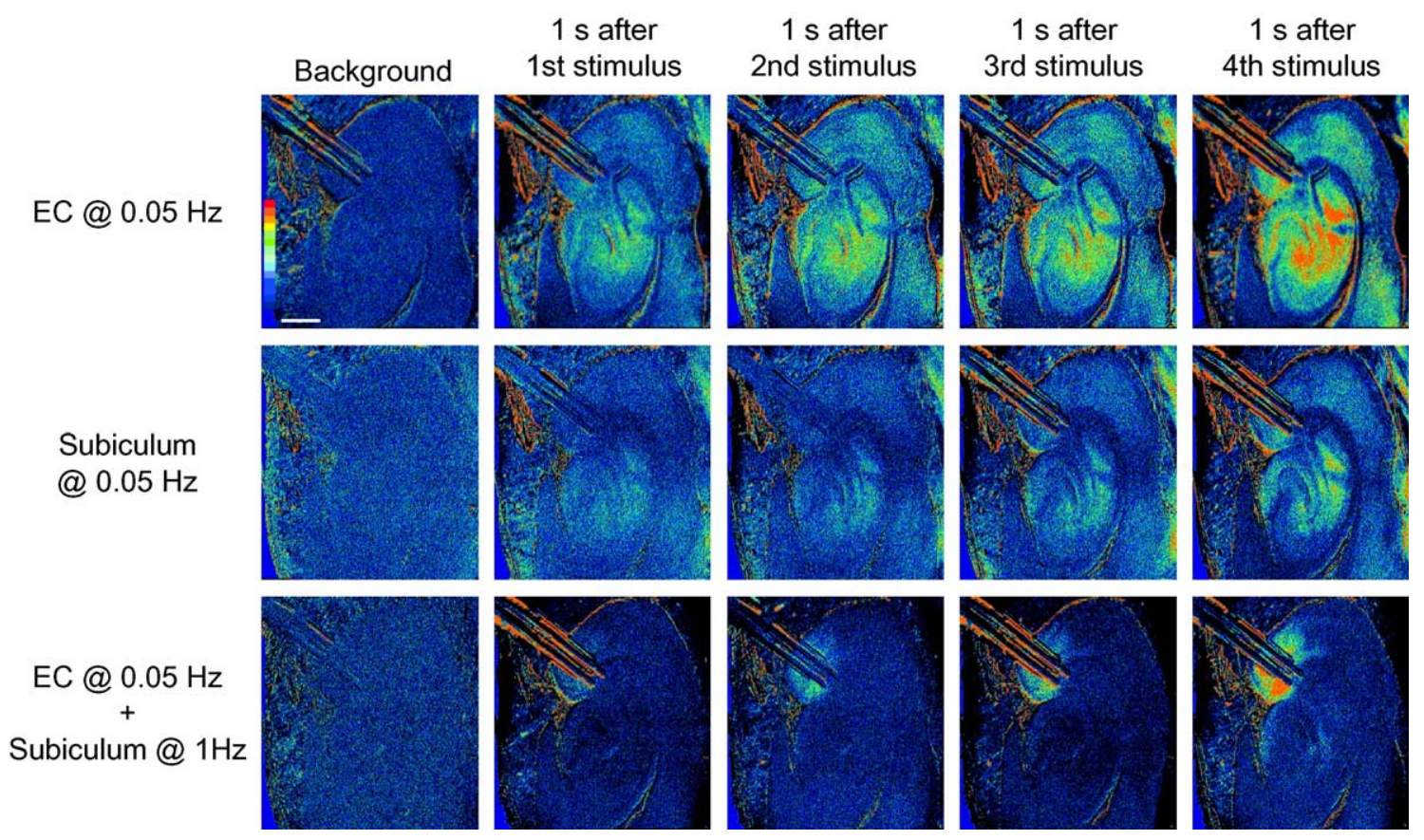

Fig. 4. IOS changes elicited by recurrent, single-shock electrical stimuli delivered at $0.05 \mathrm{~Hz}$ in the EC deep layers (upper row), by repetitive subicular stimuli at $0.05 \mathrm{~Hz}$ (middle row), and by pairing the $0.05-\mathrm{Hz}$ stimulation of the $\mathrm{EC}$ with $1-\mathrm{Hz}$ stimuli delivered in the subiculum (lower row). IOS frames in the upper and lower rows were obtained before (Background) and $1 \mathrm{~s}$ after the 1st to 4th stimulus of two series of EC simuli; frames in the middle row represent successive frames before (background) and $1 \mathrm{~s}$ after the $1 \mathrm{st}$ to 4 th stimulus of a series of subicular stimuli. In this experiment, EC stimulation only causes IOS changes that appear first in the subiculum and then increase progressively in intensity to involve the EC and the perirhinal cortex and to propagate to the hippocampus proper ( $1 \mathrm{~s} \mathrm{after}$ the 4 th stimulus frame). In contrast, continuous $0.05 \mathrm{~Hz}$ subicular stimulation induces appreciable changes in IOSs only after the 3rd stimulus of the series. Note that when the EC train is delivered during continuous subicular stimulation at $1 \mathrm{~Hz}$, IOSs are reduced in the EC where they are restrained to the medial portion and virtually absent in the hippocampus proper. Scale bar in the background frame in the upper row $=2.5 \mathrm{~mm}$. 
In these experiments as well, low-intensity subicular stimulation at either $0.05 \mathrm{~Hz}$ (Fig. 4, Subiculum at $0.05 \mathrm{~Hz}$ ) or $1 \mathrm{~Hz}$ (data not shown) induced IOS changes that were less intense (or even absent) than those seen when similar stimulating procedures were made in the EC at high strength. Moreover, as shown in Fig. 4 (EC at $0.05 \mathrm{~Hz}+$ Subiculum at $1 \mathrm{~Hz}$ row), focal extracellular stimuli delivered at $0.05 \mathrm{~Hz}$ in the deep EC layers in conjunction with repetitive subicular stimulation at $1 \mathrm{~Hz}$ caused IOS changes that remained localized to the EC overlying the stimulating electrode, thus presumably blocking the spread of the epileptiform activity to the perirhinal cortex and the hippocampus proper. In addition, the IOS increases seen in the EC during repetitive stimulation of the subiculum were of lower amplitude than those seen under control conditions.

The percentage of the IOS increases as well as the spatial extent of the IOS changes observed in different regions of the hippocampus-EC slice following 5 consecutive stimuli delivered at 0.05 $\mathrm{Hz}$ in the $\mathrm{EC}$ in control and during repetitive subicular stimulation at $1 \mathrm{~Hz}$ are summarized in Figs. 5A and B for 8 different experiments. When these quantitative data were plotted as percentage of IOS inhibition (Fig. 5C), we found that IOSs were inhibited by 83.4 and $74.3 \%$ in the EC and subiculum and by $100 \%$ in CA3-CA1 and by $98 \%$ in the dentate gyrus. Similar findings were obtained by analyzing the reduction of the size of the different areas involved in generating IOSs (Fig. 5D). In these experiments, we also established the correlation coefficient between the reductions in IOS intensity and the decreases in IOS area size seen in EC and dentate gyrus during repetitive subicular stimulation at $1 \mathrm{~Hz}$. By using the Spearman's rank correlation test, we found that the reductions in these two parameters had $r=0.542$ in the EC and $r=0.769$ in the dentate gyrus. Hence, these results further indicate that repetitive subicular stimulation at $1 \mathrm{~Hz}$ can decrease the intensity of the IOS changes associated with EC activation as well as the spread of the synchronous (presumably epileptiform) activity from the EC to the hippocampus proper.

\section{Effects induced by repetitive subicular stimulation at different frequencies on stimulus-induced IOSs}

To date, the ability of repetitive stimulation to inhibit ictogenesis in parahippocampal slices was usually limited to frequencies up to $1 \mathrm{~Hz}$ (Barbarosie and Avoli, 1997; Barbarosie et al., 2002; Benini et al., 2003). Therefore, in 6 slices, we used different rates (i.e., from 0.2 to $10 \mathrm{~Hz}$ ) of repetitive low-strength, subicular stimulation to establish the frequency that was most effective in reducing the IOS increases that occur after recurrent, single-shock stimulation of the EC deep layers at $0.05 \mathrm{~Hz}$.

As shown in the quantitative histograms of Fig. 6, we found that the maximal reduction of the IOS increases as well as of the area of the involved limbic structures analyzed after 5 stimuli delivered in the $\mathrm{EC}$ at $0.05 \mathrm{~Hz}$ occurred during repetitive subicular stimuli delivered at $1 \mathrm{~Hz}$. In addition, repetitive stimuli delivered at 5 (and in some cases 10) $\mathrm{Hz}$ were unable to decrease significantly the IOS changes that were presumably associated with ictal synchronization. We could also confirm in these experiments that, when present, the larger inhibitory effects occurred in the CA1 and CA3 areas.

\section{Comparison of the effects induced by repetitive stimulation of subiculum or EC on the stimulus-induced IOS changes}

Finally, we tested whether similar reductions in IOS area and intensity could be induced by repetitive stimuli delivered in the EC. Therefore, in 6 experiments, we compared the effects induced by repetitive $1 \mathrm{~Hz}$ stimulation that was delivered in either the subiculum
A

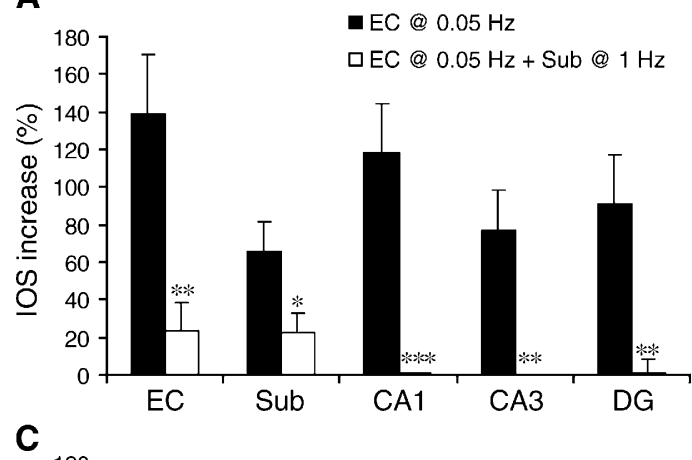

B
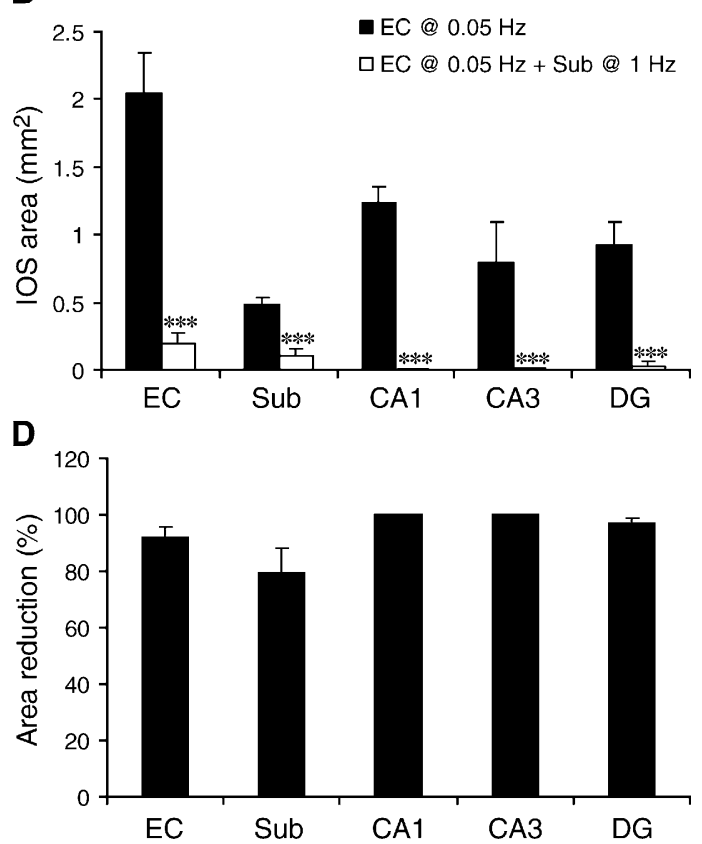

Fig. 5. Quantification of the IOS changes elicited in 10 slices treated with 4AP by $0.05-\mathrm{Hz}$ stimulation delivered in the EC deep layers under control conditions and during repetitive subicular stimuli at $1 \mathrm{~Hz}$. (A and B) Measurements of the IOS changes in terms of \% increases and area size in different regions of the brain slice; values were obtained under control conditions and during repetitive subicular stimulation at $1 \mathrm{~Hz}, 5 \mathrm{~s}$ after the 4th stimulus of the EC. (C and D) Inhibition of the $\%$ increases and of the area size exerted by $1-\mathrm{Hz}$ subicular stimulation in the same experiments shown in $\mathrm{A}$ and $\mathrm{B}$. Note that in these experiments, the maximal inhibition is seen in the CA3 and CA1 areas as well. Levels of significance: ${ }^{*} P<0.05 ;{ }^{* *} P<0.01$; and ${ }^{* * *} P<0.001$. 
A

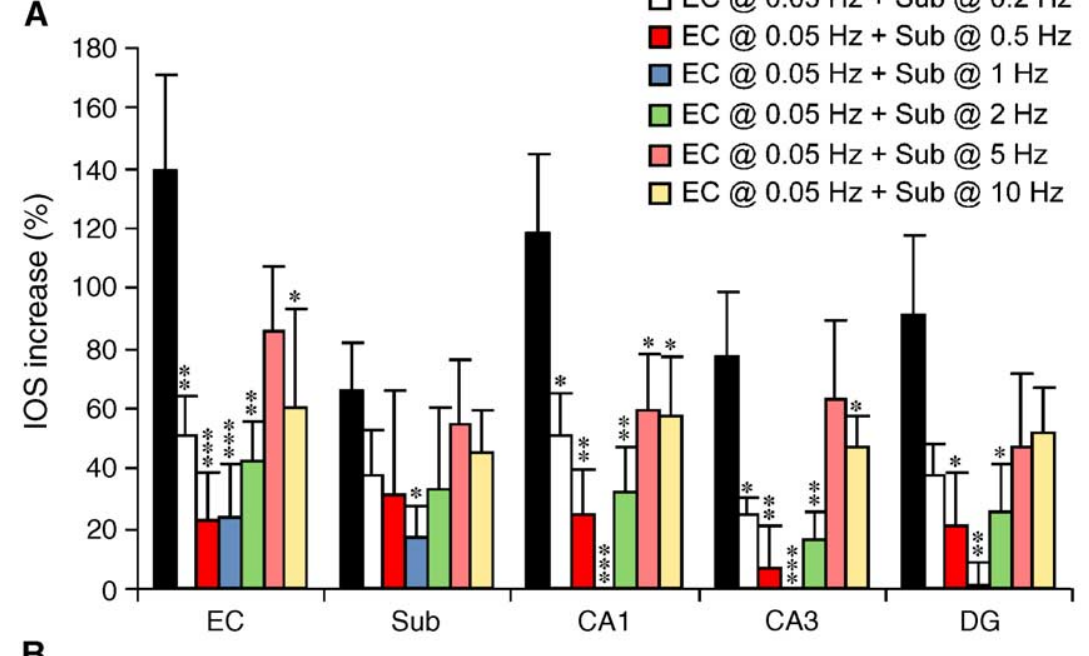

B

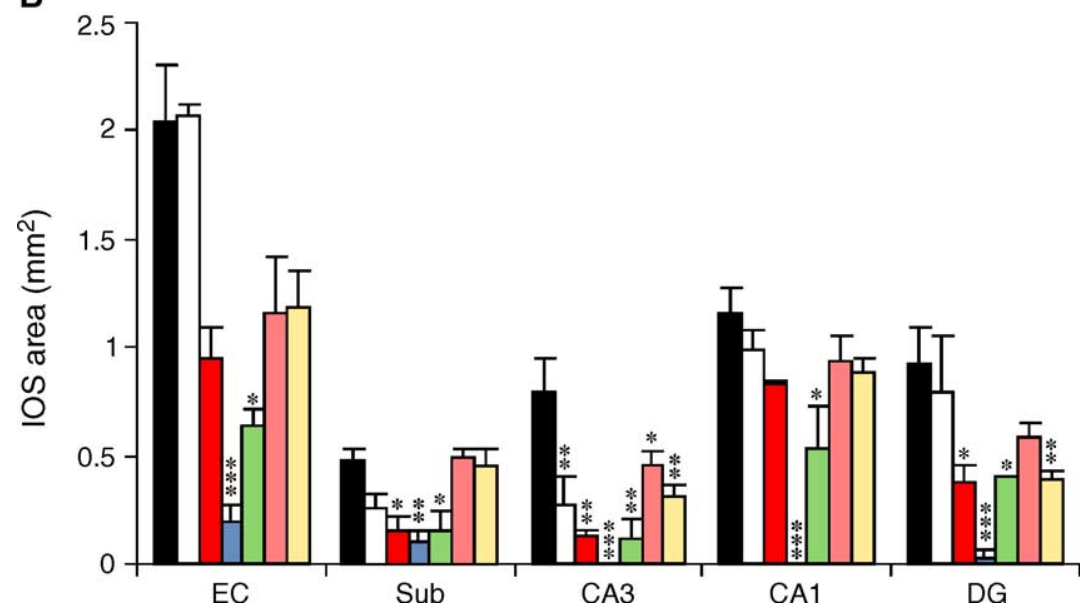

Fig. 6. Quantification of the effects induced by repetitive stimuli delivered in the subiculum at rates ranging between 0.2 and $10 \mathrm{~Hz}$ on the IOS changes that were seen following 4 recurrent stimuli at $0.05 \mathrm{~Hz}$ in the EC deep layers. Note that maximal reduction of the IOS increases as well as of the involved areas occur during repetitive subicular stimulation delivered at $1 \mathrm{~Hz}$, while these inhibitory effects are reduced by increasing the rate of continuous subicular stimulation to 5 or $10 \mathrm{~Hz}$. Note also that the larger reductions are seen in the CA1 and CA3 areas of the hippocampus proper. Levels of significance: $* P<0.05$; $* * P<0.01 ;$ and $* * * P<0.001$.

or the EC on the IOS increases caused by train stimulation or $0.05 \mathrm{~Hz}$ recurrent stimulation of the EC deep layers.

As illustrated in Figs. 7A and B, the 1-Hz stimulation protocol was effective in reducing the intensity and the areas of IOS activation induced by EC train stimuli when delivered in either EC or subiculum. However, the reductions of the areas of IOS activation, and in particular those seen in the hippocampus proper, were often more pronounced with stimuli delivered in the subiculum. Similar results could also be obtained by analyzing the effects induced on the intensity and the areas of IOS activation caused by recurrent stimuli delivered in the EC at $0.05 \mathrm{~Hz}$ (Figs. 7C and D).

\section{Discussion}

The overall goal of this study was to obtain quantitative information on the inhibitory action exerted by low-frequency, repetitive stimulation of limbic networks on ictogenesis in rat brain slices treated with the convulsant drug 4AP. To this end, we exploited the ability of IOS recordings to identify both the intensity and the spatial extent of the neuronal activation within the limbic areas contained in the in vitro slice preparation. Several studies have shown that IOSs are closely related to spontaneous or stimulus-induced synchronous neuronal activity that resembles epileptiform discharges (Buchheim et al., 1999, 2000; Hochman et al., 1995; Meierkord et al., 1997). Moreover, we have reported in a previous study performed in combined EC-hippocampus slices (D'Arcangelo et al., 2001) that stimulus-induced IOSs observed during 4AP treatment appear first in the EC (and sometimes simultaneously in subiculum), and only later they become evident in the DG, CA3, and CA1. This evidence supports the view that limbic seizures in patients presenting with temporal lobe epilepsy (Rutecki et al., 1989; Spencer and Spencer, 1994; Bartolomei et al., 2001) and in animal models (Bragdon et al., 1992; Dreier and Heinemann, 1991; Nagao et al., 1996; Avoli et al., 1996) originate in EC and then propagate to the hippocampus proper. Similar temporal patterns of IOS activation were obtained in this study following brief train of stimuli at $10 \mathrm{~Hz}$ or recurring single-shock stimulation at $0.05 \mathrm{~Hz}$. Moreover, we have found that: (i) the 
A

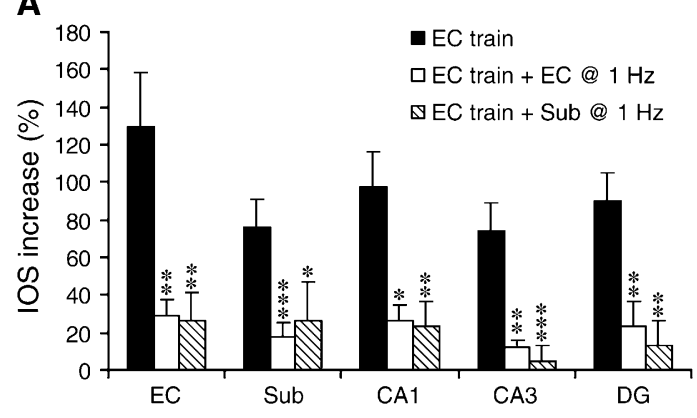

C

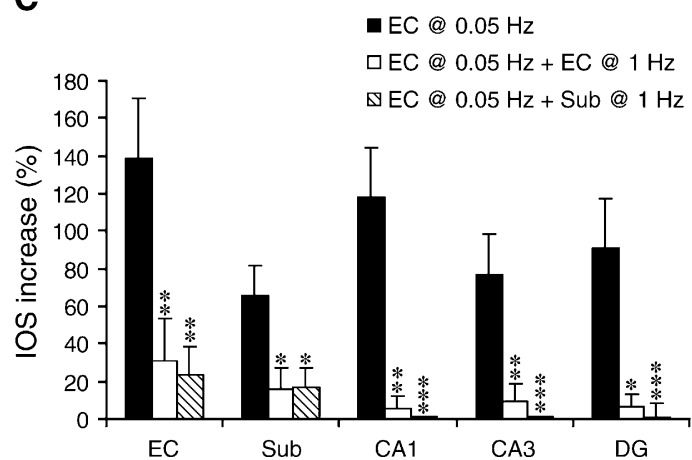

B

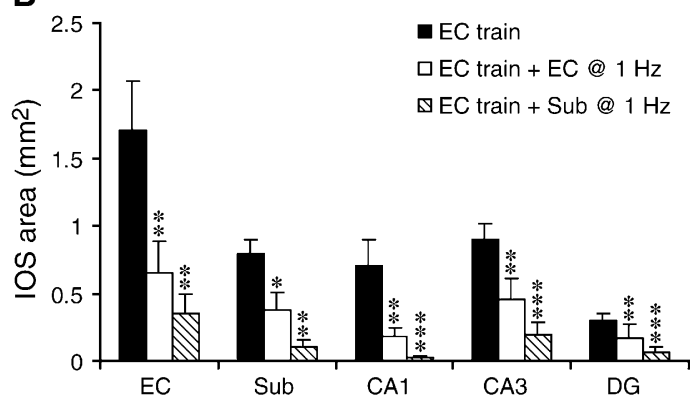

D

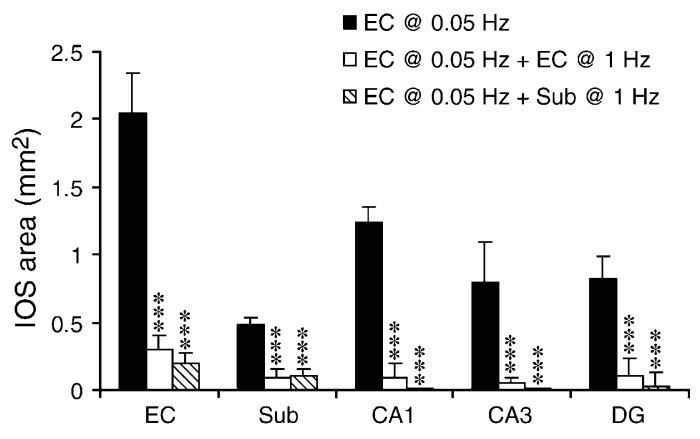

Fig. 7. Comparison of the inhibitory effects induced by repetitive 1-Hz stimulation delivered in either the subiculum or the EC on the IOS increases caused by train stimulation (A and B) or $0.05-\mathrm{Hz}$ recurrent stimulation $(\mathrm{C}$ and $\mathrm{D})$ of the EC deep layers. In A and $\mathrm{B}$, IOS changes in terms of \% increases and area size were measured in different regions of the brain slice under control conditions and during repetitive subicular or EC stimulation at $1 \mathrm{~Hz}, 10 \mathrm{~s}$ after the stimulus train delivered in the EC. In C and D, IOSs in terms of \% increases and area size were measured under control conditions and during repetitive EC or subicular stimulation at $1 \mathrm{~Hz}, 5 \mathrm{~s}$ after the 4th stimulus of the EC series. Note that, in most of the limbic areas, the increase in IOS and the area size were reduced to a larger extent during repetitive stimuli delivered in the subiculum. Levels of significance: ${ }^{*} P<0.05 ; * * P<0.01 ;$ and $* * * P<0.001$.

inhibitory effects on the IOS changes presumably associated with ictogenesis are evident in all limbic areas during 1-Hz stimulation of the subiculum; (ii) the stimulation frequency (within a range of 0.2 to $10 \mathrm{~Hz}$ ) that is most effective in reducing IOS changes corresponds to $1 \mathrm{~Hz}$; and (iii) remarkable inhibitory effects can be induced by repetitive stimuli delivered in both subiculum and EC, even though the former site appears to be more effective.

In addition, to confirm that stimulus-induced ictal-like responses analyzed during 4AP application with field potential recordings are reduced in duration and eventually abolished by repetitive subicular stimuli at frequencies ranging from 0.2 to $1 \mathrm{~Hz}$ (Barbarosie and Avoli, 1997; Benini et al., 2003), we have found here that IOS generated in response to trains of stimuli or recurrent single shock at $0.05 \mathrm{~Hz}$ in the EC are reduced in both intensity and amount of activated areas during repetitive subicular stimulation at $1 \mathrm{~Hz}$. These effects were evident in all limbic regions comprised in our slice preparation, but appeared maximally expressed in terms of normalized inhibition in the hippocampus proper (and specifically in the CA3 subfield). Hence, this evidence may imply that repetitive stimulation not only controls the initiation of ictal discharges within the EC network, but it may also influence the propagation of these events to the hippocampus proper.

These data are in keeping with those observed in mouse EChippocampal slices during application of $4 \mathrm{AP}$ or $\mathrm{Mg}^{2+}$-free medium (Barbarosie and Avoli, 1997; Barbarosie et al., 2002). Moreover, Benini et al. (2003) have reported a similar inhibitory action on 4AP-induced parahippocampal ictogenesis during repetitive stimulation of the lateral-basolateral nuclei of the amygdala at $0.5-1 \mathrm{~Hz}$ in a rat combined hippocampus-EC-amygdala slice preparation. It has also been shown that CA3-driven interictal activity, whenever capable of reaching the EC, can exert similar inhibitory effects on the generation of spontaneous ictal discharges (Barbarosie and Avoli, 1997; Benini et al., 2003; Bragdon et al., 1992; cf., for review, de Curtis and Avanzini, 2001). However, this condition was not met in our study since the main CA3 outputs, which run through the Schaffer collateral system, were always cut at the beginning of the experiment.

The patterns of repetitive stimulation used in our study to inhibit ictogenesis were delivered at intensities that were unable to elicit ictal-like responses (when analyzed with field potential recordings) or IOS changes similar to those seen following brief stimulus train or single-shock stimuli at $0.05 \mathrm{~Hz}$. This characteristic is in line with what obtained in studies performed in patients presenting with intractable temporal lobe epilepsy where deepbrain or transcranial magnetic stimulation produced both inhibitory or excitatory effects, depending on the stimulus intensity applied. For instance, Yamamoto et al. (2002) have reported an exacerbation of epileptic activity when the stimulus intensity was high (e.g., $7.5 \mathrm{~mA}$ ), while inhibitory effects were obtained by using low stimulus intensity (e.g., $<0.5 \mathrm{~mA}$ ).

The frequency of the repetitive stimuli applied to the parahippocampal networks is an important parameter for reducing and eventually abolishing ictal synchronization. Significant reductions of the IOS intensity and of the areas involved in generating IOSs became evident at $0.5 \mathrm{~Hz}$ and maximal effects were always observed with $1 \mathrm{~Hz}$. Indeed, this value approximates that of the rate of occurrence of CA3-driven interictal events. Hence, we are inclined to propose that repetitive electrical stimuli may restrain the generation of ictal responses in the EC by depressing the function of $\mathrm{GABA}_{\mathrm{A}}$ receptor-mediated synchronization that in the 4AP in vitro 
model is associated with large $\left[\mathrm{K}^{+}\right]_{\mathrm{o}}$ elevations capable of initiating ictal discharges (reviewed by Avoli et al., 2002). Such a mechanism has been identified in mouse slices treated with 4AP where it contributes to the inhibitory action of CA3-driven interictal activity on ictogenesis (Barbarosie et al., 2002). However, it must be emphasized that the $1-\mathrm{Hz}$ value identified as optimal frequency of stimulation in the present study may not be valid in the clinical contest where higher frequencies have been reported to be effective in controlling seizures.

Alternatively, low-frequency stimulation may suppress ictogenesis by causing long-term depression of synaptic transmission within the limbic networks contained in our slice preparation. Long-term depression is induced by low-frequency electric stimulation in the hippocampus (Dudek and Bear, 1992), visual cortex (Kirkwood et al., 1993), and motor cortex (Hess and Donoghue, 1996). However, in our experiments, the length of repetitive stimulation was usually shorter than what required for LTD induction (i.e., 4-10 $\mathrm{min}$ in our experiments vs. $15 \mathrm{~min}$ in studies made on long-term depression) and ictal discharges reappeared within less than 2 min upon termination of the stimulating procedure. Therefore, these differences suggest that the ability of repetitive stimuli to control ictogenesis may be unrelated to LTD.

We have also explored whether the inhibitory action exerted by repetitive stimulation depends on the structure that is stimulated. In particular, we compared stimuli delivered in the subiculum (which is the gating structure for hippocampal outputs) with those in the EC deep layers. Our findings show that stimulation of both areas could control the IOS increases associated with ictogenesis; however, we have found that stimuli delivered in subiculum were the most effective. Hence, these observations support the view that this control mechanism is activity dependent, but also pathway specific. Although little is known about how subicular neurons activate EC deep-layer cells, the more marked suppression of ictal synchronization seen when the stimulating electrode is placed in the subiculum may be due to their ability to desynchronize GABAergic interneurons within the EC network. Once more, this condition could better mimic the findings observed during CA3-driven interictal discharge (Barbarosie and Avoli, 1997). In contrast, low-frequency stimulation of the EC should involve presumably both the afferences from the hippocampus and the local circuit between deep and superficial layers of the EC.

In conclusion, our study supports the view that low-frequency repetitive stimulation of limbic networks can control ictogenesis in the EC as well as the spread of this activity to the hippocampus and, perhaps in the in vivo condition, to para- and extra-limbic areas, thus leading to generalization of the epileptic activity. Since CA3 cells are functionally and/or anatomically damaged in mesial temoral lobe epileptic patients, we propose that, at least in the in vitro slice preparation used here, the repetitive stimulation may constitute a compensatory mechanism for the down-regulation of hippocampal outputs consequent to mesial temporal lobe sclerosis (Avoli et al., 2002).

\section{Acknowledgments}

This study was supported by grants from the FIRB-MIUR (Ministero dell'Istruzione, Università e Ricerca) and the Canadian Institutes of Health Research (Grant MT-8109). We thank Ms. T. Papadopoulos for secretarial assistance.

\section{References}

Andrew, R.D., MacVicar, B., 1994. Imaging cell volume changes and neuronal excitation in the hippocampal slice. Neuroscience 62 , $371-383$

Andrew, R.D., Jarvis, C.R., Obeidat, A.S., 1999. Potential sources of intrinsic optical signals imaged in live brain slices. Methods 8, 185-196.

Avoli, M., Barbarosie, M., Locke, A., Nagao, T., Lopantsev, V., Khling, R., 1996. Synchronous GABA-mediated potentials and epileptiform discharges in the rat limbic system in vitro. J. Neurosci. 16, 3912-3924.

Avoli, M., D’Antuono, M., Louvel, J., Kohling, R., Biagini, G., Pumain, R., D'Arcangelo, G., Tancredi, V., 2002. Network and pharmacological mechanisms leading to epileptiform synchronization in the limbic system. Prog. Neurobiol. 68, 167-207.

Barbarosie, M., Avoli, M., 1997. CA3-driven hippocampal-entorhinal loop controls rather than sustains in vitro limbic seizures. J. Neurosci. 17, 9308-9314.

Barbarosie, M., Louvel, J., D’Antuono, M., Kurcewicz, I., Avoli, M., 2002. Masking synchronous GABA-mediated potentials controls limbic seizures. Epilepsia 43, 469-1479.

Bartolomei, F., Wendling, F., Bellanger, J., Regis, J., Chauvel, P., 2001. Neural networks involved in temporal lobe seizures: a nonlinear regression analysis of SEEG signals interdependencies. Clin. Neurophysiol. 112, 1746-1760.

Benini, R., D’Antuono, M., Pralong, E., Avoli, M., 2003. Involvement of amygdala networks in epileptiform synchronization in vitro. Neuroscience $120,75-84$.

Bragdon, A.C., Kojima, H., Wilson, W.A., 1992. Suppression of interictal bursting in hippocampus unleashes seizures in entorhinal cortex: a proepileptic effect of lowering $\left[\mathrm{K}^{+}\right]_{\mathrm{o}}$ and raising $\left[\mathrm{Ca}^{2+}\right]_{\mathrm{o}}$. Brain Res. $590,128-135$.

Buchheim, K., Schuchmann, S., Siegmund, H., Gabriel, H.J., Heinemann, U., Meierkord, H., 1999. Intrinsic optical signal measurements reveal characteristic features during different forms of spontaneous neuronal hyperactivity associated with ECS shrinkage in vitro. Eur. J. Neurosci. 11, 1877-1882.

Buchheim, K., Schuchmann, S., Siegmund, H., Weissinger, F., Heinemann, U., Meierkord, H., 2000. Comparison of intrinsic optical signals associated with low $\mathrm{Mg}^{2+}$ - and 4-aminopyridine-induced seizure-like events reveals characteristic features in adult rat limbic system. Epilepsia 41, 635-641.

D’Arcangelo, G., Tancredi, V., Avoli, M., 2001. Intrinsic optical signals and electrographic seizures in the rat limbic system. Neurobiol. Dis. 8, 993-1005

de Curtis, M., Avanzini, G., 2001. Interictal spikes in focal epileptogenesis. Prog. Neurobiol. 63, 541-567.

Dodt, H.U., D’Arcangelo, G., Pestel, E., Zieglgänsberger, W., 1996. The spread of excitation in neocortical columns visualized with infrareddarkfield videomicroscopy. NeuroReport 7, 1553-1558.

Dreier, J.P., Heinemann, U., 1991. Regional and time dependent variations of low $\mathrm{Mg}^{2+}$ induced epileptiform activityin rat temporal cortex slices. Exp. Brain Res. 87, 581-596.

Dudek, S.M., Bear, M.F., 1992. Homosynaptic long-term depression in area CA1 of hippocampus and effects of $N$-methyl-D-aspartate receptor blockade. Proc. Natl. Acad. Sci. U. S. A. 89, 4363-4367.

Hess, G., Donoghue, J.P., 1996. Long-term depression of horizontal connections in rat motor cortex. Eur. J. Neurosci. 8, 658-665.

Hochman, D., Baraban, S., Owens, J., Schwartzkroin, P., 1995. Dissociation of synchronization and excitability in furosemide blockade of epileptiform activity. Science 270, 99-102.

Holthoff, K., Witte, O., 1996. Intrinsic optical signal in rat neocortical slices measured with near-infrared dark-field microscopy reveal changes in extracellular space. J. Neurosci. 16, 2740-2749.

Kirkwood, A., Dudek, S.M., Gold, J.T., Aizenman, C.D., Bear, M.F., 1993. Common forms of synaptic plasticity in the hippocampus and neocortex in vitro. Science 260, 1518-1521. 
MacVicar, B., Hochman, D., 1991. Imaging of synaptically evoked intrinsic optical signals in hippocampal slices. J. Neurosci. 11, 1458-1469.

Meierkord, H., Schuchmann, S., Buchheim, K., Heineman, U., 1997. Optical imaging of low $\mathrm{Mg}^{2+}$-induced spontaneous activity in combined rat entorhinal cortex-hippocampal slices. NeuroReport 8, 1857-1861.

Menkes, D.L., Gruenthal, M., 2000. Slow-frequency repetitive transcranial magnetic stimulation in a patient with focal cortical dysplasia. Epilepsia $41,240-242$.

Nagao, T., Alonso, A., Avoli, M., 1996. Epileptiform activity induced by pilocarpine in the rat hippocampal-entorhinal slice preparation. Neuroscience 72, 399-408.

Rutecki, P.A., Grossman, R.G., Armstrong, D., Irish-Loewen, S.J., 1989. Electrophysiological connections between the hippocampus and entorhinal cortex in patients with complex partial seizures. J. Neurosurg. 70, 667-675.

Spencer, S.S., Spencer, D.D., 1994. Entorhinal-hippocampal interactions in temporal lobe epilepsy. Epilepsia 35, 721-727.

Tergau, F., Naumann, U., Paulus, W., Steinhoff, B.J., 1999. Low-frequency repetitive transcranial magentic stimulation improves intractable epilepsy. Lancet 353, 2209.
Velasco, M., Velasco, F., Velasco, A.L., Boleaga, B., Jimenez, F., Brito, F., Marquez, I., 2000a. Subacute electrical stimulation of the hippocampus blocks intractable temporal lobe seizures and paroxysmal EEG activities. Epilepsia 41, 158-169.

Velasco, A.L., Velasco, M., Velasco, F., Menes, D., Gordon, F., Rocha, L., Briones, M., Marquez, I., 2000b. Subacute and chronic electrical stimulation of the hippocampus on intractable temporal lobe seizures: preliminary report. Arch. Med. Res. 31, 316-328.

Velasco, M., Velasco, F., Velasco, A.L., 2001. Centromedian-thalamic and hippocampal electrical stimulation for the control of intractable epileptic seizures. J. Clin. Neurophysiol. 18, 495-513.

Vonck, K., Boon, P., Achten, E., De Reuck, J., Caemaert, C., 2002. Longterm amygdalo-hippocampal stimulation for refractory temporal lobe epilepsy. Ann. Neurol. 52, 556-565.

Yamamoto, J., Ikeda, A., Satow, T., Takeshita, K., Takayama, M., Matsuhashi, M., Matsumoto, R., Ohara, S., Mikuni, N., Takahashi, J., Miyamoto, S., Taki, W., Hashimoto, N., Rothwell, J.C., Shibasaki, H., 2002. Low-frequency electric cortical stimulation has an inhibitory effect on epileptic focus in mesial temporal lobe epilepsy. Epilepsia 43 , $491-495$. 